\title{
LA INTELIGENCIA EMOCIONAL EN LA GESTIÓN PEDAGÓGICA DEL DOCENTE.
}

\section{INTRODUCCIÓN}

Evidentemente las emociones que se generan en cada estudiante por la dinámica académica actual y familiar favorecerán o limitarán el aprendizaje, en consecuencia urge que el docente asuma el compromiso ético de brindar lecciones de vida a sus estudiantes por lo cual debe estar capacitado para hablar y trabajar el asunto de las emociones para el desarrollo de tales competencias, lo cual crea la urgencia de repensar en el entrenamiento para la formación de los docentes con el fin de ofrecerle herramientas que le permita abordar este proceso para su bienestar y el de sus estudiantes. En este contexto se desarrolla esta investigación que precisa el escenario de la Inteligencia Emocional con las razones que obligan a los centros de estudios primarios, secundarios y universitarios a replantear su hacer como formadoras considerando el mundo de las emociones y de la inteligencia emocional, toda vez que difícilmente un docente que no muestre equilibrio en sus emociones puede detectar y ayudar a sus estudiantes en este aspecto, "nadie enseña lo que no se sabe" dice un proverbio chino y esto se ajusta perfectamente, por los planteles educativos deben examinar si con el proceso de formación del docente, se está fortaleciendo su perfil personal en el marco de la Inteligencia Emocional. El planteamiento de la Inteligencia Emocional como estrategia determinante del éxito académico apunta fundamentalmente a una notabilidad práctica en sus dimensiones social y pedagógica.

\section{DESARROLLO}

LA PROPUESTA, "Estrategia para el perfeccionamiento de la inteligencia emocional en los docente Universitario. Es urgente la formación en competencias socio-emocionales de los docentes a través de la aplicación de un plan de entrenamiento para esto se propone a la autoridad de la institución el desarrollo de competencias emocionales en los docentes a través de la aplicación de la estrategia para el perfeccionamiento de la inteligencia emocional en los docente universitario como paso inicial para una adecuada intervención con sus estudiantes unido a esto el efecto protector de la Inteligencia Emocional frente al burnout (desgaste profesional) que sufren muchos docentes como consecuencia del estrés al que se ven sometidos en su labor profesional, esto adquiere gran importancia cuando se trata de docentes que son considerados elementos claves para promover la formación en competencias profesionales y emocionales de los estudiante que ingresan, que ya son parte y los que están por egresar de la universidad que se convierten automáticamente en la imagen de la académica hacia la colectividad.

\section{CONTENIDO DE LA PROPUESTA}

"Estrategia para el perfeccionamiento de la inteligencia emocional en los docentes de los centros de estudios. 


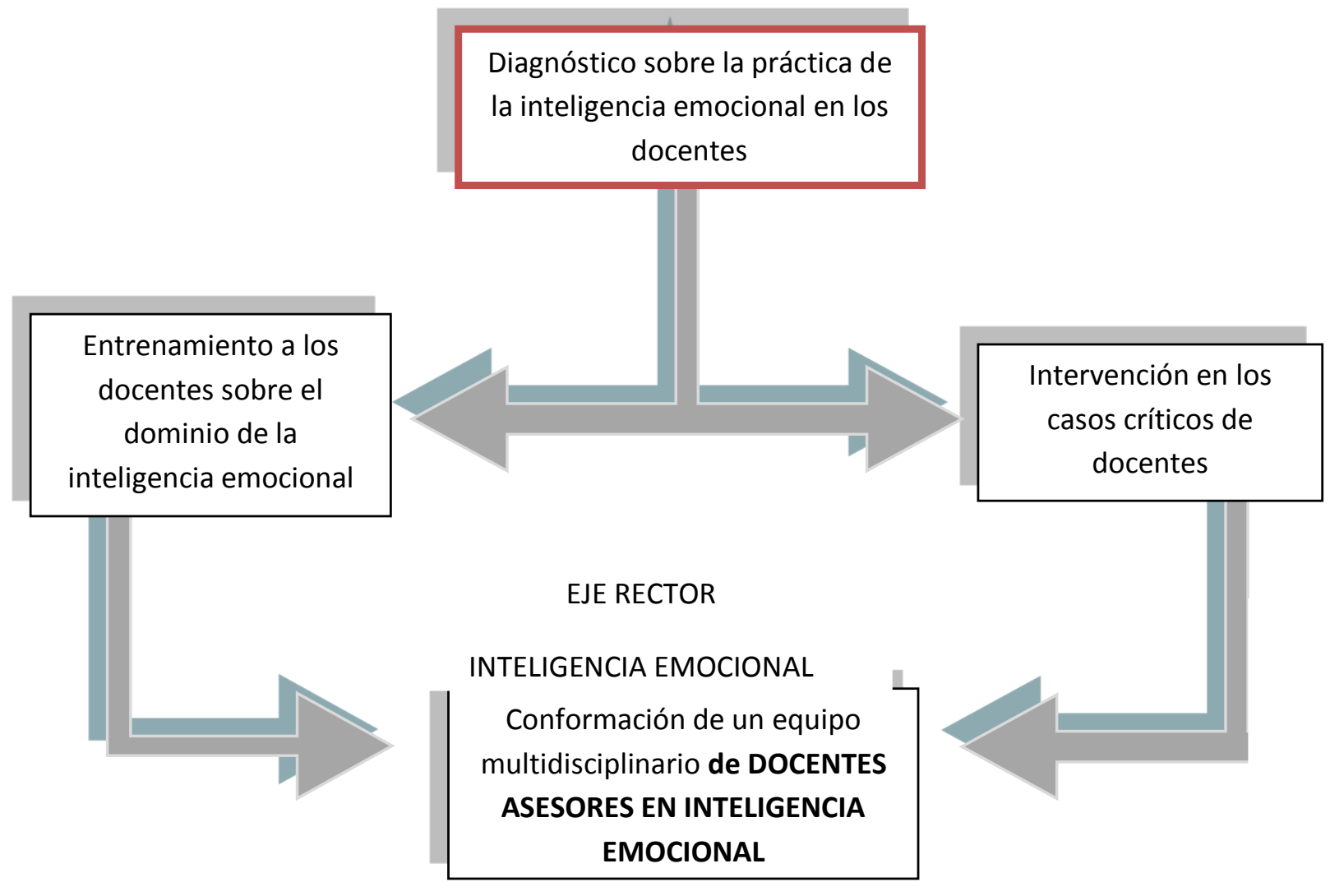

FUNDAMENTACIÓN DE LA ESTRATEGIA.- Mejorar la Inteligencia Emocional y generar el dominio de la IE con el cambio de la actitud del docente entrenándose en el Marco de las Competencias Emocionales como son, la COMPETENCIA PERSONAL, y la COMPETENCIA SOCIAL estas competencias enmarcan la Autoconciencia, Autorregulación, la motivación, la empatía, autoestima, autocontrol, dedicación, integridad, habilidad para comunicar, pericia para iniciar y aceptar cambios. Una competencia emocional es una capacidad adquirida basada en la inteligencia emocional que da lugar a un desempeño laboral-docente sobresaliente, en el núcleo de esta competencia se encuentran dos habilidades, la empatía (que supone la capacidad de interpretar los sentimientos ajenos) y las habilidades sociales (que nos permiten manejar diestramente esos sentimientos). Las competencias emocionales se agrupan en conjuntos, cada uno de los cuales está basado en una capacidad subyacente de la inteligencia emocional, capacidades que son vitales si las personas quieren aprender las competencias necesarias para tener éxito en su trabajo. Si carecen de habilidades sociales, por ejemplo, serán incapaces de persuadir o inspirar a los demás, de dirigir equipos o de catalizar el cambio. En caso de que tengan poca conciencia de sí mismos, por ejemplo, no serán conscientes de sus propios puntos flacos y, en consecuencia, carecerán de la suficiente confianza que sólo puede derivarse de la seguridad en la propia fortaleza. Esta estrategia, busca fundamentalmente entrenar a los docentes en Inteligencia Emocional para que adquiera los elementos necesarios para un desempeño ejemplar y dominio de la Inteligencia Emocional, sólo requieren ser fuertes en un determinado número de competencias y luego que estas se hallen dispersas en las cinco regiones de la inteligencia emocional. Dicho en 
otras palabras, son muchos los caminos que conducen a la excelencia. Las capacidades de la inteligencia emocional son las siguientes:

- Independencia: Cada persona aporta una contribución única al desempeño de su trabajo.

- Interdependencia: Cada individuo depende en cierta medida de los demás, con los que se halla unido por interacciones muy poderosas.

- Jerarquización: Las capacidades de la inteligencia emocional se refuerzan mutuamente. Por ejemplo, la conciencia de la misma persona resulta esencial para el autocontrol y la empatía; el autocontrol y la conciencia de sí mismo contribuye a la motivación, y estas cuatro capacidades resultan esenciales, a su vez, para desarrollar las habilidades sociales.

- Necesidad pero no suficiencia: Poseer una inteligencia emocional subyacente no garantiza que la gente acabe desarrollando o ejerciendo las competencias asociadas con ella, como, por ejemplo, la colaboración y el liderazgo. Factores tales como el clima que se respira en el área en que se ejerce la docencia o el interés de las personas por su trabajo también determinan si estas aptitudes acabarán manifestándose.

- Genéricas: La lista general resulta, hasta cierto punto, aplicable a todos los trabajos, pero cada profesión exige competencias diferentes. En este caso de estudio diseñada para el docente de la UNESUM Paralelo Santa Ana, como parte fundamental para el desarrollo de su gestión pedagógica aplicando la inteligencia emocional.

\section{ACTIVIDADES}

a. Diagnostico previo sobre los Cuatro pilares de la Inteligencia Emocional

Primer Pilar: Conocimiento Emocional. Entre los cuales se encuentran: Honestidad Emocional, Energía Emocional

Energía Tensa, Energía Tranquila, Cansancio Tenso, Cansancio Tranquilo

Retroinformación Emocional, Intuición Práctica.

Segundo Pilar: Aptitud Emocional. Presencia Autentica, Radio de Confianza, Descontento Constructivo,Flexibilidad y Renovación.

Tercer Pilar: Profundidad Emocional. Potencial único y Propósito, Integridad Aplicada,Influencia sin Autoridad, Compromiso, responsabilidad y Conciencia.

Cuarto Pilar: Alquimia Emocional. Flujo Intuitivo, Percepción de Oportunidades, Creación del Futuro, Cambio de tiempo reflexivo. 

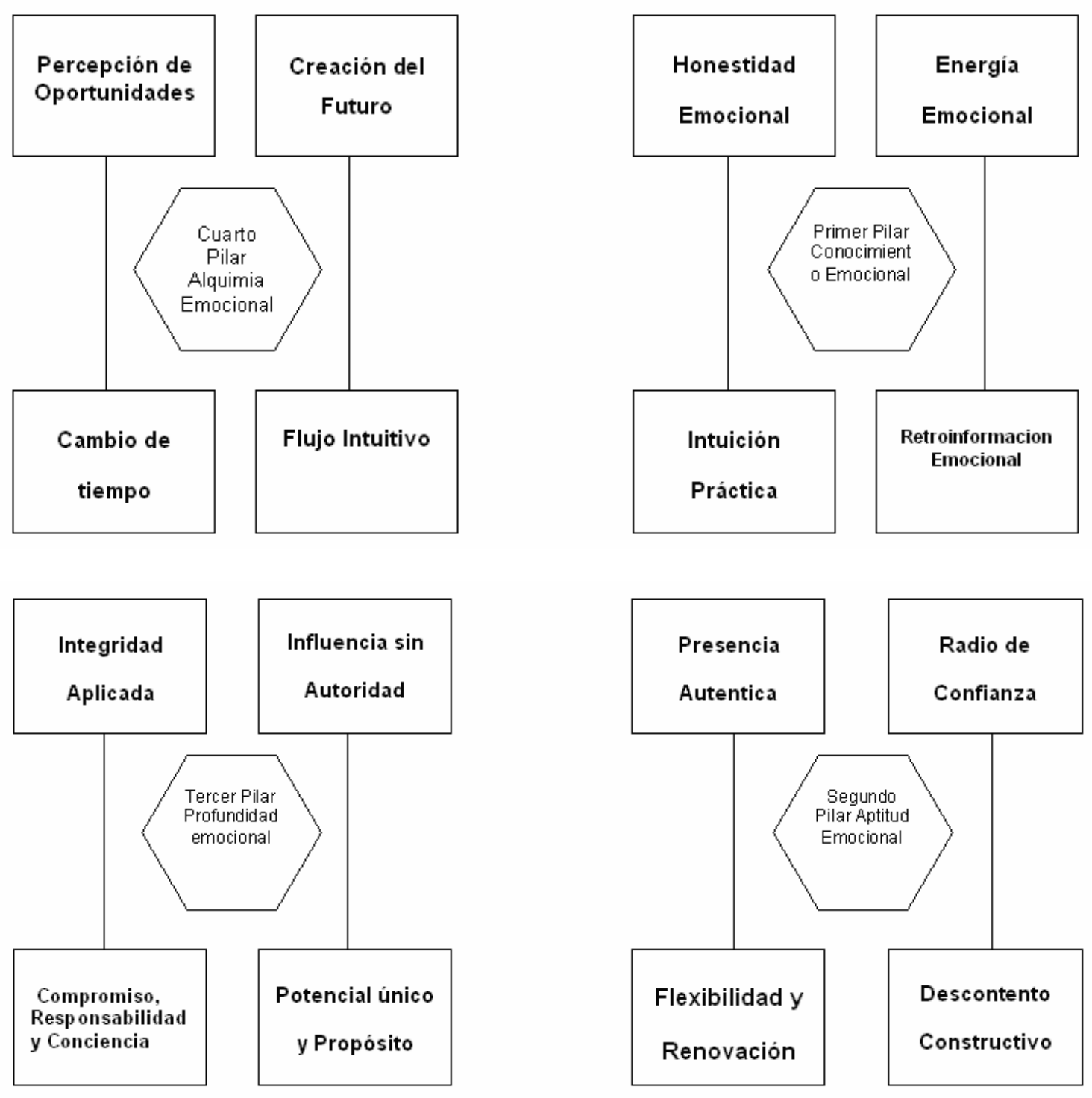

Bibliografia: Goleman, Daniel. 2007. Inteligencia Social, Tema: Habilidades Gerenciales Goleman, Daniel. 2009. La Inteligencia Emocional en la empresa http://danielgoleman.info/topics/emotional-intelligence/

Microbiografia Autora: Ing. Alexandra Verduga Pino.,Profesora Universitaria, Magister en Gerencia Educativa, Senadora de la JCl / Osaka, Japón, Instructora Graduada en EE.UU, Consultora Internacional. Email: alexandraverduga@yahoo.com - Facebook: Alexandra Verduga Twitter: @DraVerduga - Blogger: Protocolo Tecnico - Linkedin: Alexandra Verduga 